\title{
Rúbrica para la Evaluación de la Competencia Innovación, Creatividad y Emprendimiento en máster.
}

Llanos Cuenca ${ }^{a}$, Faustino Alarcón ${ }^{b}$, Andrés Boza ${ }^{c}$, Marta Fernández-Diego ${ }^{d}$, Leonor Ruiz ${ }^{\text {e, Mari Luz Gordof, Raul Polerg, Mareva Alemany }}{ }^{\text {h }}$

a,c,d,e,f Escuela Técnica Superior de Ingeniería Informática. Universitat Politècnica de València (SPAIN)., llcuenca@omp.upv.es, aboza@omp.upv.es, marferdi@omp.upv.es, lruiz@omp.upv.es , magormon@upvnet.upv.es

b,g,h Escuela Técnica Superior de Ingeniería Industrial. Universitat Politècnica de València (SPAIN). faualva@omp.upv.es,rpoler@omp.upv.es, mareva@omp.upv.es

\begin{abstract}
Innovation has a special value for the survival and development of organizations, especially in a changing context. To develop the innovation, creativity and entrepreneurship capacities in students enhances their skills. A competency describes what training participants should be able to do at the end of the training. The competence is acquired through various learning outcomes to be achieved. Competition in innovation is closely related to the ability to propose and imple-ment creative ideas to solve problems, ability to create and maintain connections work, etc. In this article is presented a method for measuring the competence of innovation, creativity and entrepreneurship in master degree by introducing different levels of scope.
\end{abstract}

Keywords: Rubric, competence, innovation, evaluation

\begin{abstract}
Resumen
La innovación tiene un especial valor para la supervivencia y el desarrollo de las organizaciones, más aún en un contexto de permanente cambio. El desarrollo de la capacidad de innovación en los estudiantes favorece su capacidad de inserción en el mundo laboral. Una competencia describe lo que los participantes en la formación deben ser capaces de hacer al final de dicha formación. La competencia es adquirida a través de varios resultados de aprendizaje a alcanzar. La competencia en innovación está estrechamente relacionada con la capacidad para proponer y aplicar ideas creativas para resolver problemas, capacidad de crear y mantener conexiones de trabajo,
\end{abstract}


etc. En este artículo ser presenta un método para medir la competencia de innovación creatividad y emprendimiento en el nivel de máster mediante la introducción de diferentes niveles de alcance a través de una rúbrica.

Palabras clave: Rúbrica, competencia, innovación, evaluación

\section{Introducción}

El presente artículo se enmarca dentro del proyecto de innovación y mejora educativa RECICRE, centrado en la definición e implementación de mecanismos de evaluación para la adquisición de la dimensión competencial de la Universitat Politècnica de València, DC4- innovación, creatividad y emprendimiento y del proyecto OAICE orientado al diseño de objetos de aprendizaje para favorecer la adquisición de la dicha competencia. La rúbrica definida puede ser transferible a otras asignaturas y otros centros de la UPV. Se ha trabajado sobre los resultados de aprendizaje que deben ser alcanzados, diferenciando grado y máster, y definido rúbricas de evaluación que permiten especificar el sistema de evaluación de la adquisición de competencias, procurando de esta forma una evaluación continua del alcance de los resultados de aprendizaje y de las competencias previstas en la asignatura o la materia objeto de evaluación. El proyecto se ha desarrollado en colaboración con el proyecto Europeo IDEA Tempus el cual tiene como objetivo mejorar la integración de los conocimientos en el área interdisciplinaria de Ingeniería, Diseño y Negocios en la educación superior desde una perspectiva industrial, la mejora de la innovación, así como mejorar las relaciones educación- industria

La motivación que lleva a la presente propuesta es contribuir a sistematizar mecanismos de evaluación que garanticen la adquisición de la dimensión competencial innovación, creatividad y emprendimiento.

\section{Desarrollo de la innovación}

El objetivo general del proyecto se concretó en varios objetivos detallados. Cada uno de los objetivos detallados se ha llevado a cabo mediante una o varias actividades desarrolladas por los investigadores del proyecto. La secuenciación de las actividades ha permitido realizar interacciones entre ellas para completar el objetivo deseado.

Las actividades uno y dos siguieron el proceso de revisión bibliográfica para analizar los resultados de aprendizaje relacionados con la competencia de innovación para grado y máster. Se llevó a cabo a través de Google Scholar (scholar.google.es) y Scopus; también incluyendo la lista preliminar de resultados de aprendizaje del Instituto de Ciencias de la Innovación (Ice, 2013) y los resultados del proyecto (Tempus, 2014). Para el desarrollo de la rúbrica (actividad tres) y siguiendo la similitud que estas tienen con los modelos de 
madurez, se aplicó la metodología definida en (Cuenca et al. ,2013). Se identificaron las áreas clave a medir (en este caso los resultados de aprendizaje) y la descripción de cada uno de los niveles de alcance, para terminar con la definición completa de la rúbrica. El desarrollo de la actividad tres (definición de rúbrica) puede llevar a replantearse la definición y/o redacción de los resultados de aprendizaje, así como los resultados de la actividad cuatro (validación) pueden implicar una mejor definición de la rúbrica. Por último la revisión y cierre permite valorar los resultados y plantear acciones de mejora en la validación y/o definición de la rúbrica.

\section{Resultados}

Los resultados obtenidos en el proyecto están directamente relacionados con las actividades desarrolladas y han servido para evaluar el cumplimiento de las mismas. Se ha diseñado la rúbrica para evaluar los RA de máster asociados a la competencia de innovación, creatividad y emprendimiento. Hemos establecido la relación entre competencias, resultados de aprendizaje y objetos/actividades de aprendizaje (Fig. 1).

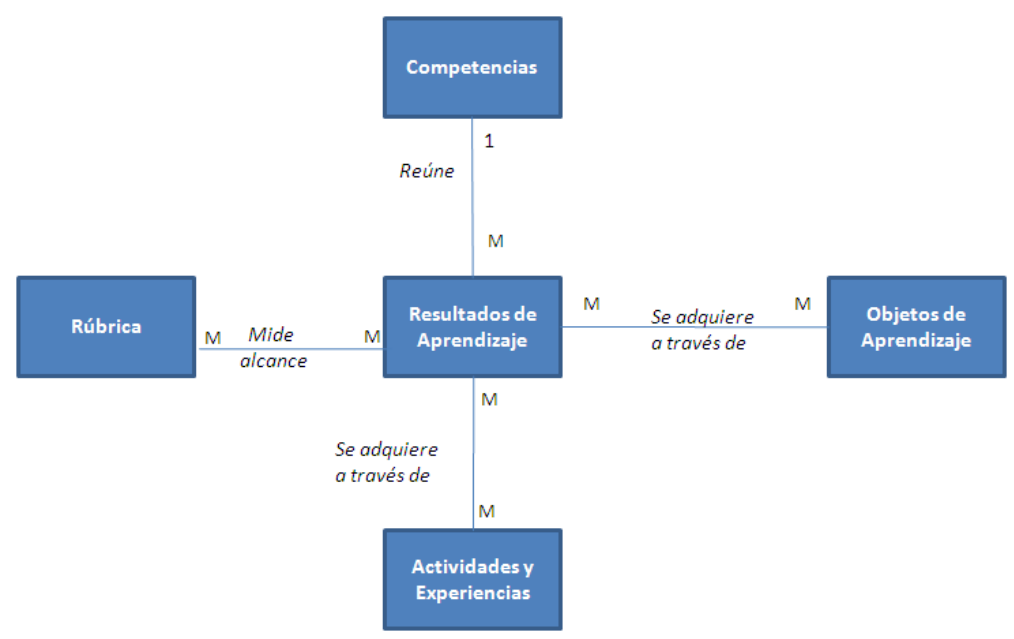

Fig. 1 Elementos asociados a los resultados de aprendizaje en las competencias

Se ha clasificado los resultados de aprendizaje asociados a la competencia objeto de estudio en diferentes perspectivas como son, creatividad, emprendimiento, integración y previsión Bapat et al., 2014) (Las dos últimas englobadas en innovación).

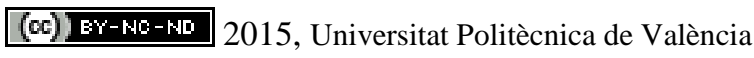

Congreso In-Red (2015) 
Creatividad: (GI) Generación de ideas: Llegar a una variedad de enfoques para la resolución de problemas. (CT) Pensamiento Crítico: identificar lógicamente cómo los diferentes enfoques posibles son fuertes y débiles, y el análisis de estos juicios. (S / R) Síntesis / Reorganización: Cómo encontrar una mejor manera de acercarse a los problemas a través de la síntesis y la reorganización de la información. (CPR) Resolución de problemas creativamente: Uso de nuevas ideas para resolver problemas como líder.

Emprendimiento: (IP) La identificación del problema: Precisar la naturaleza real y la causa de los problemas y las dinámicas que subyacen en ellas. (SI) Buscando Mejora: constantemente buscando maneras que uno puede mejorar la propia organización. (GI) Recopilación de información: Identificación de fuentes útiles de información y la recolección y la utilización de la información que es esencial. (IT) Pensamiento independiente: Pensar diferente, aunque a veces esto pueda ir en contra de la opinión popular. (TS) Comprensión tecnológica: La comprensión y la utilización de la tecnología para mejorar los procesos de trabajo.

Integración: (OI) apertura a las ideas: La voluntad de escuchar las sugerencias de los demás y para probar nuevas ideas. (RO) Orientación de Investigación: La observación de la conducta de los demás, leer mucho, y mantener su mente abierta a ideas y soluciones de otros. Leer y hablar con la gente en los campos relacionados para descubrir innovaciones o las tendencias actuales en el campo. (C) Colaboración: Trabajar con los demás y la búsqueda de las opiniones de los demás para llegar a una solución creativa. (ENW) Participar en otros Intereses relacionados: Completo y búsqueda de información de otros campos y ámbitos de la vida para encontrar nuevos enfoques para situaciones.

Previsión: (PS) Percibir: Reconociendo los cambios importantes que se producen en un sistema o predecir con exactitud cuándo podrían ocurrir. (CE) Evaluación de consecuencias a largo plazo: concluir lo que un cambio en los sistemas dará lugar a largo plaz. (V) Visión: Desarrollar una imagen de un ideal estado de trabajo de una organización. (MF) Gestionar el Futuro: La evaluación de las direcciones y los riesgos futuros basados en las fortalezas actuales y futuras, debilidades, oportunidades y amenazas.

La tabla 1 muestra la rúbrica diseñada.

Tabla 2. Rúbrica para la Evaluación de la Competencia Innovación, Creatividad y Emprendimiento en Máster

\begin{tabular}{|c|c|c|c|c|c|c|}
\hline 잉 & & $\begin{array}{l}\text { spectiva IResultado } \\
\text { aprendizaje }\end{array}$ & Nivel 1 & Nivel 2 & Nivel 3 & Nivel 4 \\
\hline$\underset{\substack{\frac{k}{\alpha} \\
\frac{\mathrm{m}}{\mathrm{x}}}}{\mathrm{v}}$ & CRS & $\begin{array}{l}\text { Adoptar enfoques } \\
\text { creativos en relación al } \\
\text { contenido propio de la } \\
\text { situación y al modo de }\end{array}$ & $\begin{array}{lr}\text { Se } & \text { ofrecen } \\
\text { pocas ideas } \\
\text { innovadoras } 0 \\
\text { no } \quad \text { son }\end{array}$ & $\begin{array}{l}\text { Suficientes } \\
\text { enfoques } y \\
\text { adaptados a bien } \\
\text { situación planteada }\end{array}$ & $\begin{array}{lr}\text { Adopción } & \text { de } \\
\text { enfoques } & \text { creativos } \\
\text { al } & \text { contenido } \\
\text { adecuado } & \text { de la la }\end{array}$ & $\begin{array}{lr}\text { Adopta } & \text { enfoques } \\
\text { creativos } & \text { bien } \\
\text { adaptados } & y \\
\text { ampliados } & \text { en } \\
\text { el }\end{array}$ \\
\hline
\end{tabular}


Llanos Cuenca, Faustino Alarcón, Andrés Boza , Marta Fernández-Diego, Leonor Ruize, Mari Luz Gordo, Raul Poler, Mareva Alemany

\begin{tabular}{|c|c|c|c|c|c|}
\hline & realización. & $\begin{array}{l}\text { adecuadas la } \\
\text { para la la } \\
\text { situación } \\
\text { estudiada }\end{array}$ & $\begin{array}{l}\text { pero sin especificar } \\
\text { correctamente el } \\
\text { modo de ejecución }\end{array}$ & \begin{tabular}{|l} 
situación y un \\
correcto \\
planteamiento del \\
modo de realización
\end{tabular} & tiempo \\
\hline CT & $\begin{array}{l}\text { Evaluar críticamente los } \\
\text { datos y sacar } \\
\text { conclusiones. }\end{array}$ & $\begin{array}{lr}\text { Se realiza } & \text { un } \\
\text { análisis } & \text { y } \\
\text { evaluación } & \\
\text { limitado de los } \\
\text { datos sin } \\
\text { obtener } \\
\text { conclusiones }\end{array}$ & $\begin{array}{l}\text { Evaluación critica } \\
\text { correcta de los } \\
\text { datos pero limitada } \\
\text { y unas conclusiones } \\
\text { claras pero escasas }\end{array}$ & $\begin{array}{lr}\text { Evaluación rritica } \\
\text { correcta de los datos } \\
\text { definiendorr unas } \\
\text { conclusiones } & \text { claras } \\
\text { y detalladas } & \end{array}$ & $\begin{array}{l}\text { Evaluación crítica } \\
\text { de calidad, } \\
\text { obteniéndose un } \\
\text { gran número de } \\
\text { conclusiones } \\
\text { claras y detalladas }\end{array}$ \\
\hline CRS & $\begin{array}{l}\text { Introducir soluciones } \\
\text { singulares o punteras } \\
\text { que mejoran varios } \\
\text { aspectos o ámbitos de } \\
\text { actuación. }\end{array}$ & $\begin{array}{l}\text { No se lleva a } \\
\text { cabo la } \\
\text { adaptación de } \\
\text { soluciones a } \\
\text { las situaciones } \\
\text { planteadas }\end{array}$ & $\begin{array}{lr}\text { Limitado manejo de } \\
\text { soluciones } & 0 \\
\text { escaso interés } & 0 \\
\text { novedad de estas } & \end{array}$ & \begin{tabular}{l}
\multicolumn{3}{l}{ Correcta adaptación } \\
de ideas y \\
soluciones \\
novedosas que \\
permiten apreciar las \\
mejoras
\end{tabular} & $\begin{array}{lr}\text { Alto número } & \text { de } \\
\text { soluciones } & \\
\text { singulares } & \text { que } \\
\text { resuelven y } & \text { se } \\
\text { adaptan a } & \text { un } \\
\text { número } & \text { alto } \\
\text { también } & \text { de } \\
\text { ámbitos } & \text { de } \\
\text { actuación } & \end{array}$ \\
\hline GI & $\begin{array}{l}\text { Utilizar métodos } \\
\text { específicos para mejorar } \\
\text { la creatividad. }\end{array}$ & $\begin{array}{l}\text { No utiliza } \\
\text { métodos } \\
\text { específicos } \\
\text { para mejorar la } \\
\text { creatividad }\end{array}$ & $\begin{array}{l}\text { Utiliza métodos para } \\
\text { mejorar la } \\
\text { creatividad pero no } \\
\text { son específicos }\end{array}$ & $\begin{array}{lr}\text { Utiliza } & \text { métodos } \\
\text { específicos } & \text { y } \\
\text { adecuados } & \text { para } \\
\text { mejorar } & \text { la } \\
\text { creatividad } & \end{array}$ & $\begin{array}{l}\text { Utiliza métodos } \\
\text { específicos para } \\
\text { mejorar la } \\
\text { creatividad y estos } \\
\text { logran muy buenos } \\
\text { resultados }\end{array}$ \\
\hline CRS & $\begin{array}{l}\text { Utilizar métodos y } \\
\text { soluciones adecuadas a } \\
\text { la innovación. }\end{array}$ & $\begin{array}{l}\text { Los métodos y } \\
\text { soluciones } \\
\text { empleados no } \\
\text { son adecuados } \\
\text { a la innovación } \\
\text { o no utiliza } \\
\text { métodos y } \\
\text { soluciones } \\
\text { enfocados a la } \\
\text { innovación }\end{array}$ & $\begin{array}{l}\text { Utiliza métodos y } \\
\text { soluciones a la } \\
\text { innovación si bien } \\
\text { estos podrían } \\
\text { ampliarse y no son } \\
\text { específicamente } \\
\text { adecuados a la } \\
\text { innovación }\end{array}$ & $\begin{array}{l}\text { Utiliza métodos y } \\
\text { soluciones y estos } \\
\text { son adecuados a la } \\
\text { innovación }\end{array}$ & $\begin{array}{l}\text { Utiliza profusos y } \\
\text { evolucionados } \\
\text { métodos y } \\
\text { soluciones y estos } \\
\text { son perfectamente } \\
\text { adecuados a la } \\
\text { innovación }\end{array}$ \\
\hline SR & $\begin{array}{lr}\text { Construir } & \text { el } \\
\text { conocimiento científico } & \\
\text { acerca de los problemas } \\
\text { (proyectos) } \\
\text { ingeniería, con de } \\
\text { propósito de solucionar } \\
\text { eficientemente los } \\
\text { problemas en el } \\
\text { contexto determinado. }\end{array}$ & $\begin{array}{l}\text { Se lleva a cabo } \\
\text { la observación } \\
\text { o recogida de } \\
\text { datos que } \\
\text { supone, } \\
\text { esencialmente, } \\
\text { resolver } \\
\text { problemas de } \\
\text { definición, } \\
\text { medición y } \\
\text { registro. } \\
\text { Considera } \\
\text { opciones o } \\
\text { posibilidades. }\end{array}$ & $\begin{array}{lr}\text { Se } & \text { elaboran } \\
\text { hipótesis. } & \text { Se } \\
\text { establece } & \text { la } \\
\text { evidencia } & \text { empírica } \\
\text { que } & \text { supone, } \\
\text { esencialmente, } \\
\text { resolver problemas } \\
\text { de control. }\end{array}$ & $\begin{array}{lr}\text { Tras las fases de } \\
\text { observación, } \\
\text { recogida de } & \text { datos, } \\
\text { hipótesis } & \text { y } \\
\text { evidencias } & \\
\text { verificables } & \text { se } \\
\text { establecen } & \\
\text { conclusiones. } & \end{array}$ & $\begin{array}{l}\text { Tras las fases de } \\
\text { observación, } \\
\text { recogida de datos } \\
\text { y evidencias, se } \\
\text { establecen } \\
\text { conclusiones y se } \\
\text { documenta de } \\
\text { manera clara y } \\
\text { repetible. }\end{array}$ \\
\hline CT & $\begin{array}{l}\text { Adoptar un punto de } \\
\text { vista crítico y autónomo }\end{array}$ & $\begin{array}{ll}\text { No } & \text { se } \\
\text { argumenta } & \text { de }\end{array}$ & $\begin{array}{l}\text { En ocasiones se } \\
\text { adopta en los }\end{array}$ & $\begin{array}{l}\text { En la mayoría de las } \\
\text { ocasiones se adopta }\end{array}$ & $\begin{array}{l}\text { Siempre adopta un } \\
\text { punto de vista }\end{array}$ \\
\hline
\end{tabular}

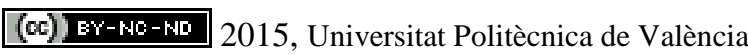

Congreso In-Red (2015) 
Rúbrica para la Evaluación de la Competencia Innovación, Creatividad y Emprendimiento en máster

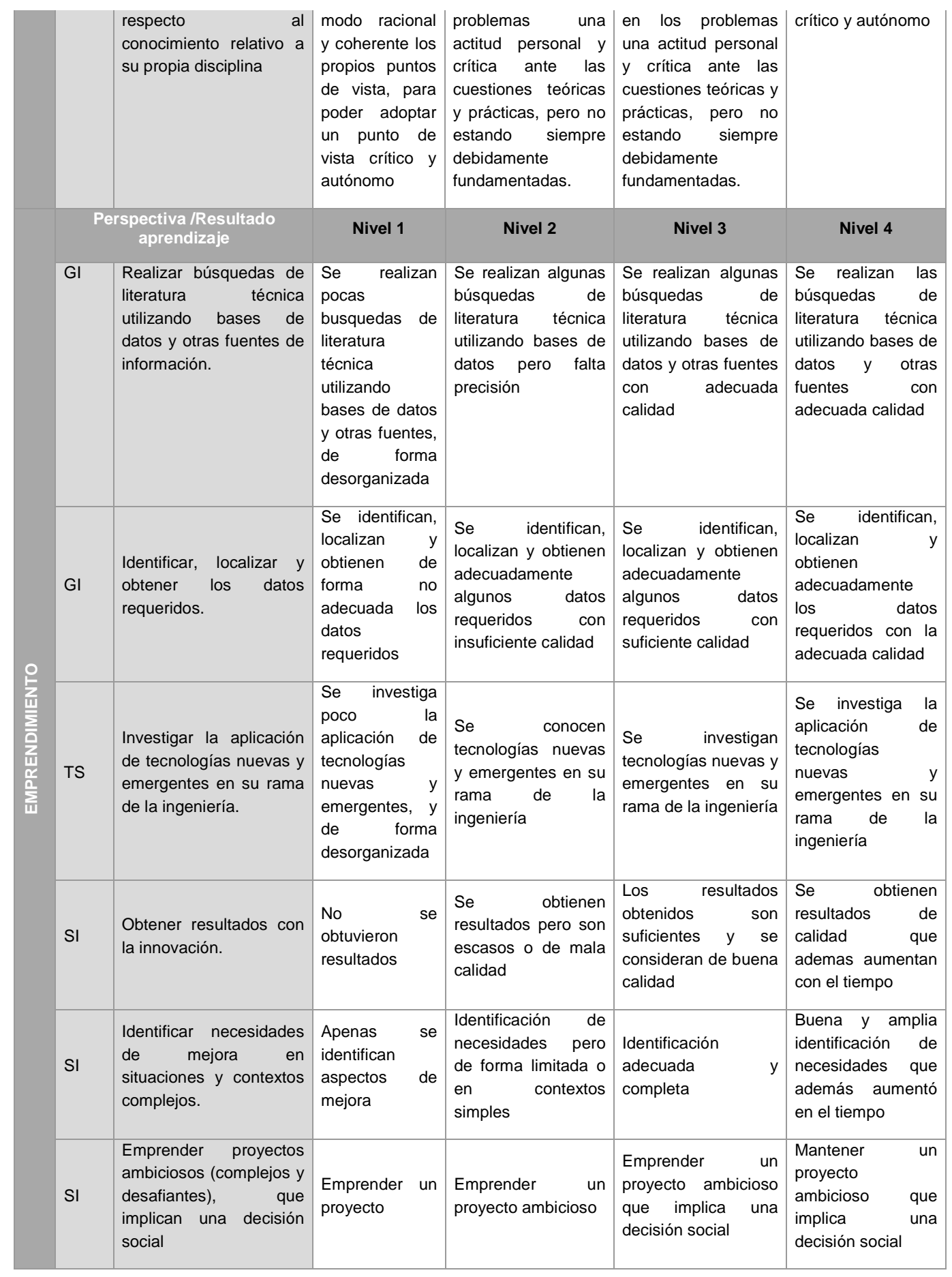

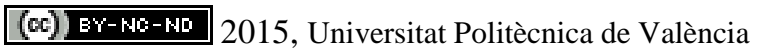

Congreso IN-RED (2015) 
Llanos Cuenca, Faustino Alarcón, Andrés Boza , Marta Fernández-Diego, Leonor Ruize, Mari Luz Gordo, Raul Poler, Mareva Alemany

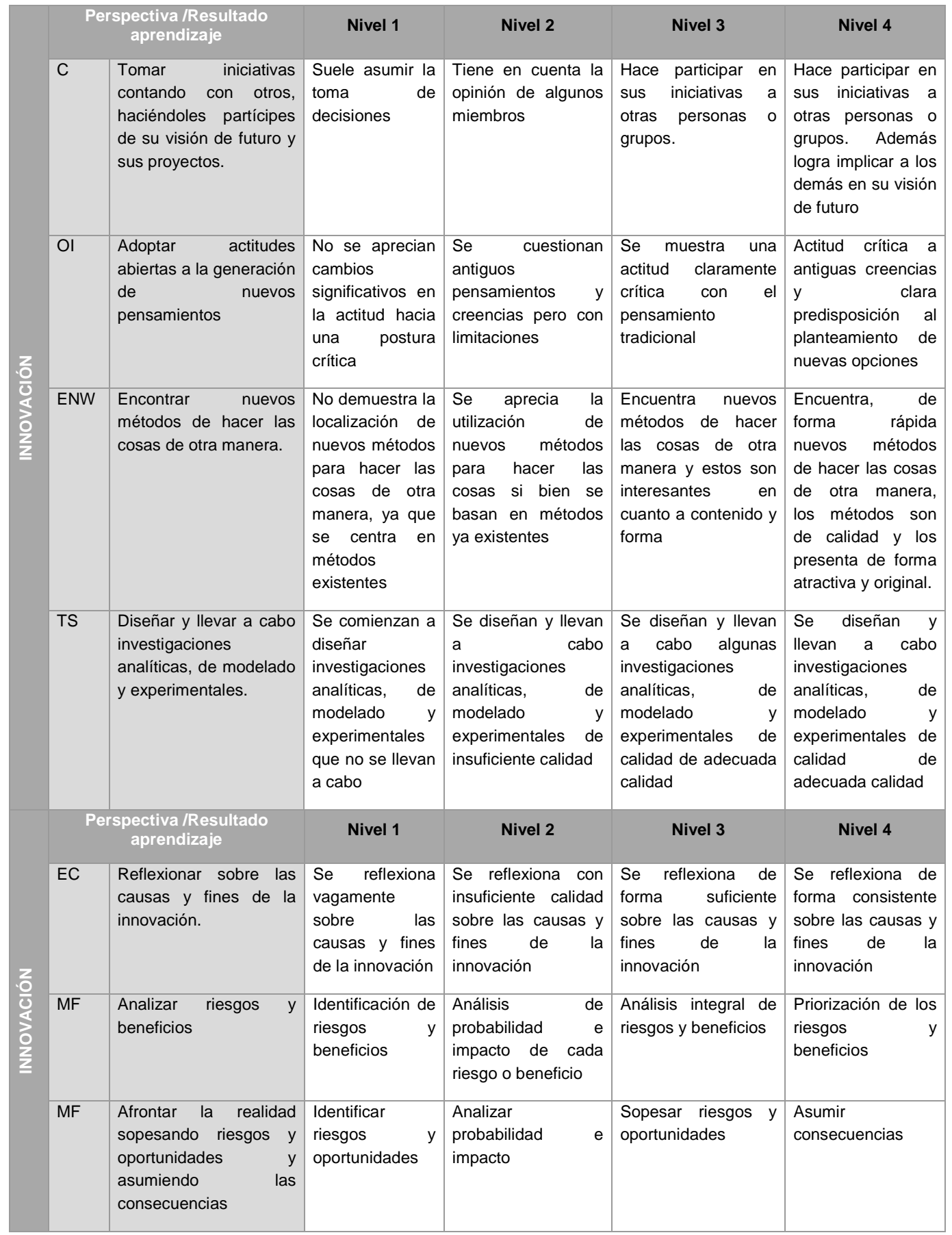

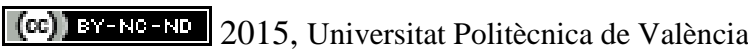

Congreso In-Red (2015) 
Aspectos importantes a considerar en la definición/aplicación de la rúbrica son:

- Se deben evitar solapes entre perspectivas/competencias

- El número de resultados de aprendizaje debe ser adecuado. Hay que analizar si el número es adecuado para balancear entre una valoración lo más completa posible y la complejidad de la misma.

- Adecuado número de niveles que facilite la aplicación.

- Alcanzar un determinado nivel puede medirse cualitativa o cuantitativamente.

- No es obligatorio analizar todos los resultados de aprendizaje, solo aquellos que se hayan considerado en una determinada actividad/experiencia.

- La rúbirca debe ser conocida por el alumno

- En la cálculo final de alcance de la competencia, se considera relevante la posibilidad de asignar pesos a cada resultado de aprendizaje, y a cada perspectiva, en lugar de calcular una media para obtener el grado de alcance. Esta valoración puede ser diferente para cada asignatura

\section{Conclusiones}

La rúbrica facilita la calificación del desempeño de los estudiantes, en áreas que son complejas de evaluar, a través de un conjunto de criterios graduados que permiten valorar el aprendizaje, los conocimientos y/o competencias logradas por el estudiante.

La principal ventaja de esta técnica para los estudiantes es que les muestra los diferentes niveles de logro que pueden alcanzar en un trabajo, proporcionando los aspectos que deben cumplir para alcanzar niveles más altos de calificación. Por otra parte, para el profesorado les permite una evaluación objetiva, justa e imparcial mediante una escala que mide las habilidades y desempeño de los estudiantes.

La estrategia de innovación seguida en el proyecto es altamente transferible al abordar una de las competencias genéricas y no está centrada en el caso particular de una asignatura.

Por último conviene destacar la importancia de desarrollar adecuados objetos de aprendizaje y actividades/experiencias que faciliten al alumno la adquisición de los resultados de aprendizaje asociados a la competencia.

\section{Agradecimientos}

La investigación se ha llevado a cabo en el marco de los proyectos de innovación para la mejora educativa (PIME/2013/A/016/B) 'RECICRE - Rúbrica para la evaluación de la competencia de innovación y mejora educativa y el proyecto OAICE (Objetos de aprendizaje para la Competencia Innovación, Creatividad y Emprendimiento.) 
Llanos Cuenca, Faustino Alarcón, Andrés Boza , Marta Fernández-Diego, Leonor Ruize, Mari Luz Gordo, Raul Poler, Mareva Alemany

\section{Referencias}

ADAMS K., 2005 The source of Innovation and Creativity. A paper commissioned by the national center on education and the economy for the new commission on the skills of the American workforce.

AMERICAN FEDERATION OF TEACHERS (AFT), National Council on Measurement in Education (NCME), \& National Education Association (NEA). (1990). Standards for teacher competence in educational assessment of students. Washington, DC: American Federation of Teachers.

BAPAT 2014 Technical Report. http://www.chsbs.cmich.edu/leader_model/assess.htm

BLACK, P., \& WILIAM, D. (1998). Assessment and classroom learning. Assessment in Education: Principles, Policy and Practice, 5(1), 7-74. CrossRef

BRAUN H., KANJEE A., BETTINGER E., AND MICHAEL KREMEr Improving Education Through Assessment, Innovation, and Evaluation. ISBN: 0-87724-058-2 by the American Academy of Arts and Sciences

BRUIN, T. DE., M. ROSEMANN, R. FREEZE, U. KULKARNI, Understanding the main phases of developing a maturity assessment model, in: Proceedings of the 16th Australasian Conference on Information Systems, Sydney, Australia, 2005, pp. 1-10.

CUENCA L., BOZA A., ALEMANY MME, TRIENEKENS J.. (2013) Structural elements of coordination mechanisms in collaborative planning processes and their assessment through maturity models: Application to a ceramic tile company. Computers in Industry 64 (2013) 898-911

CUENCA L., MARILUZ GORDO, MARTA FERNÁNDEZ-DIEGO, LEONOR RUIZ, M.M.E. ALEMANY, ANGEL ORTIZ. Chapter 10 "Measuring competencies in higher education". Sustainable Learning in Higher

DE BRUIN, T., ROSEMANN, M., FREEZE, R., \& KULKARNI, U. (2005). Understanding the main phases of developing a maturity assessment model. In Proceedings of the 16th Australasian Conference on Information Systems, Sydney, Australia, 2005, pp. 1-10.

GOMEZ P.; AGUIRRE M.P.; POSSO, F. Y GARCÍA G. (2002). Matriz de Valoración. EDUTEKA. http://www.eduteka.org/MatrizValoracion.php3

GOODRICH H. (1997). Understanding Rubrics. Educational Leadership, 54(4), 14-17

ICE 2014; Dimensiones Competenciales UPV Report Interno. ICE-Universitat Politècnica de València.

IDEA TEMPUS, 2014 Inter Disciplinary Education Agenda http://tempus-idea.org.il/idea_wp/

INCODE, 2012 Innovation competencies development as integral part of higher education. http://www.incode-eu.eu/en/

KAIRISTO =MERNAINEÄ,, T, \& NUOTIO, J. (2011). On the definition of innovation competencies. In I. Torniainen, S. Mahlamäki-Kultanen, P. Nokelainen, \& I. Paul (Eds.),

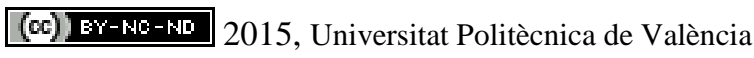

Congreso In-Red (2015) 
Rúbrica para la Evaluación de la Competencia Innovación, Creatividad y Emprendimiento en máster

Innovations for competence management conference proceedings. Series C, reports and other current publications. Part 83. Lahti: Lahti University of Applied Sciences, Esa Print.

KEEVES, J. P. (Ed.). (1997). Educational research, methodology and measurement: An international handbook (2nd ed.). New York, NY: Pergamon.

LOONEY, J. W. (2009). Assessment and innovation in education. OECD Education Working Papers, No. 24, OECD Publishing. Retrieved from http://dx.doi.org/10.1787/222814543073

LOPEZ J.L. (2002). Uso de rúbricas generalizadas para evaluar conocimientos en la técnica didáctica Aprendizaje Basado en Problemas. Documento en internet www.mty.itesm.mx/rectoria/dda/rieee/html/2002.htm [

MARIN-GARCIA J.A., PÉREZ-PEÑALVER M.J., WATTS F.(2013) How to assess innovation competence in services: The case of university students. Dirección y Organización 50 (2013) 48-62

MARKWELL ET AL, 2003 Improving teaching and learning in universities. Business/Higher education round table, issue 18, 2003

NAVARRO J.P; ORTELLS M.J,; MARTÍ M. Las rúbricas de evaluación como instrumento de aprendizaje entre pares. giac.upc.es/JAC10/09/Doc_69.pdf

NCME, 1990 American Federation of Teachers (AFT), National Council on Measurement in Education (NCME), and National Education Association (NEA). 1990. Standards for Teacher Competence in Educational Assessment of Students.Washington, DC: American Federation of Teachers.

UNESCO, 2000 UNESCO 2000b. Status and Trends 2000: Assessing learning achievement. Paris:

WATTS F., MARIN-GARCIA J.A., GARCÍA CARBONELL A., AZNAR-MAS L. Validaton of a rubric to assess innovation competence. Working Papers on Operations Management. Vol 3, № 1 (6170) ISSN: 1989-9068 